\title{
The effect of optimizers in fingerprint classification model utilizing deep learning
}

\author{
Farah F. Alkhalid \\ Control and Systems Engineering Department, University of Technology, Iraq
}

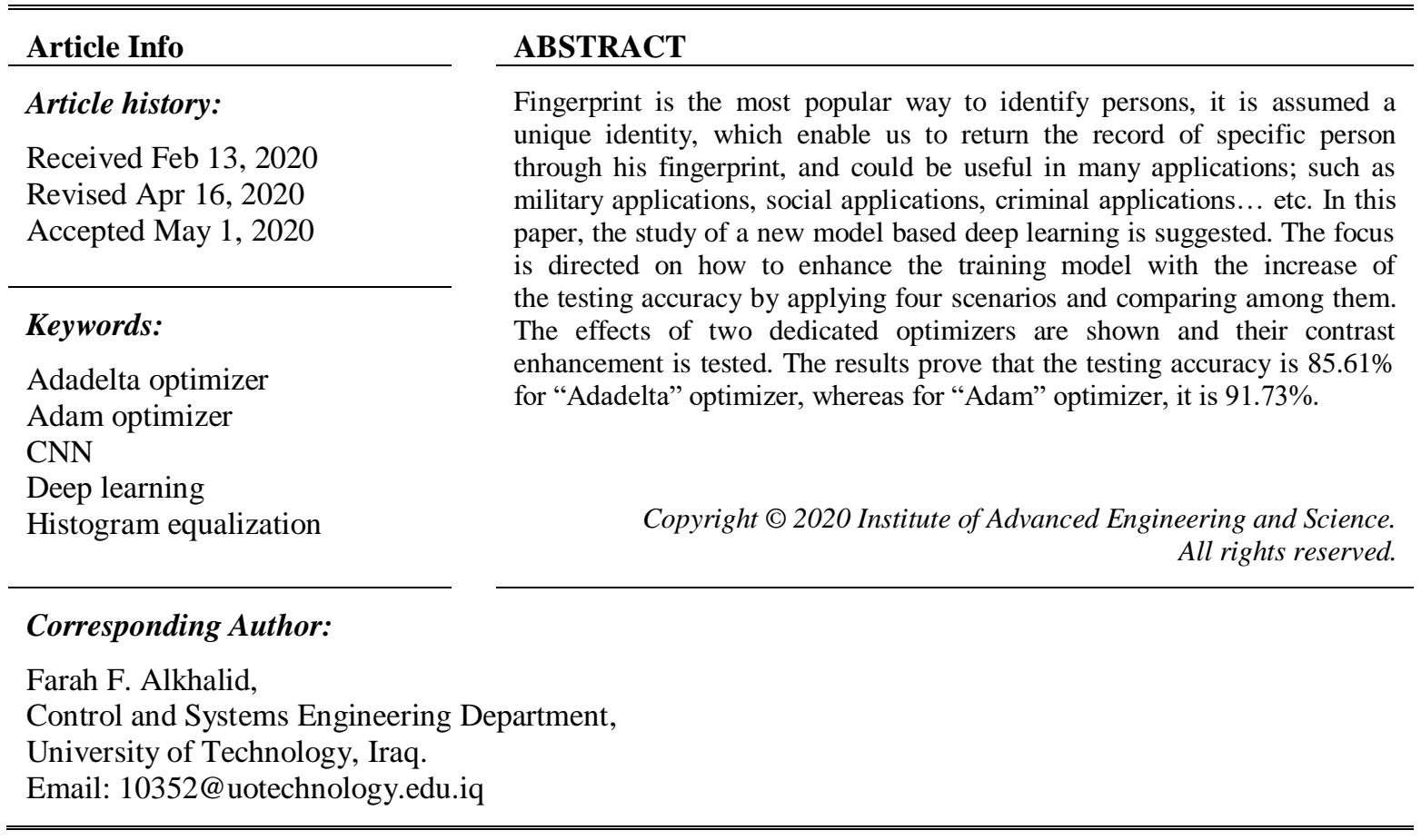

\section{INTRODUCTION}

The fingerprint is considered one of the most important methods used to identify people, especially it is unique to each person, and cannot be doubled, where people information can be retrieved by relying on the fingerprint. But this print may be latent and not clear, so by using computer vision in many fields of image processing for example improve the intensity of grey image and increase the contrast of image. On the other hand, the propelled deep learning capacities, and especially convolutional neural systems (CNN) specifically, are essentially propelling the best in class in PC vision and example acknowledgment. The deep $\mathrm{CNN}$ is an organically propelled variation of multilayer perceptron and speaks to a common deep learning engineering. Deep learning is dynamically showing its huge in areas of valuable application [1].

In this way, researchers are inquiring about and creating Deep learning strategies that are turning out to be progressively ideal. Wong et. al $[2,3]$ proposed a multi task model to enhance latent fingerprint by increasing the contrast and denoise it based CNN with very satisfied results, Imane Hachchane et. al [4] studied the face detection using fisher vector and bag of visual words with those same CNN features with satisfied results, but didn't focus on the optimizers and their effects.

In [5] proposed a model to identify and recognize fingerprint usin CNN, they focused on the speed of training and presented a very high speed model. In [6] the authors suggested to decrease the quantity of correlations in programmed unique finger impression acknowledgment frameworks with huge databases. The mix of utilizing PC vision calculations in the picture pre-handling level expands the figuring time. In [7] proposed a new study in analyzing the effects of varing the filters on accuracy of CNN model, based classifiers using human face, fingerprint and iris for person identification.

In [8] view of examination singularities and edges relating particular focuses. As a result of lowquality pictures, it is extremely hard to get right places of solitary highlights. The creators utilized investigation edge following and bends highlights to characterize fingerprints. An AI calculation that takes a 
vigorous way to deal with the order of fingerprints is SVM. This technique has made an exceptionally exact grouping framework. The benefits of the calculation are appeared in the grouping of fingerprint into various levels. The researchers of [9] utilized a blend of the SVM calculation with the innocent Bayes technique to characterize fingerprints dependent on the quantity of center and delta focuses on fingerprint. Numerous scientists have attempted to separate particular focuses in the progression of the edges [10]. The authors of [11-13] investigated a heuristic calculation with originalities to characterize fingerprint, the disservice of these investigations isn't concentrating on improving picture quality.

Since the authors used features of idiosyncrasy centers position. Which prompts an uncommon impact on the exactness of the model. The Galton-Henry course of action plot is used to arrange fingerprint. This methodology is shown in [14]. The researchers used unrest invariant partition, by then facilitate this detachment between the Finger-Code test set with the new special imprint structure. During the game plan method, the rotate invariant division extraction occurs in relating with the planning methodology. Which presents the favored situation of speedy the time of system. The Random Forest (RF) figuring is used to manage huge w holes of multi-level issues [15].

In [16] proposed a soybean disease detection model based on CNN and weight optimizer this model had provided good results but this type of optimizer is not preferred because some variables are constrained to be integers. In [17] proposed multi bioinfomatics model it was very good model with enhnacement algorithms but it did not study the effect of optimizers on the model. In [18] studied identify the finger vein detection based CNN model with very accepted results but they focused on finger vein rather than finger print identifying.

This article targets furnishing the reader with instincts concerning the conduct of various calculations for streamlining the preparation, and shows the effect of optimizers in fingerprint dataset training accuracy and also shows the effect of increasing contrast of image using histogram equalization by four scenarios as will be shown later.

\section{ADADELTA}

Adadelta [19] is a monotonically diminishing learning rate. Rather than collecting all past squared inclinations, Adadelta limits the window of aggregated past angles to some fixed size w. Rather than wastefully putting away $w$ past squared inclinations, the total of angles is recursively characterized as a rotting normal of all past squared slopes. The running normal $\mathrm{E}[\mathrm{g} 2] \mathrm{t}$ at time step $\mathrm{t}$ at that point depends (as a portion $\gamma$ likewise to the Momentum expression) just on the past normal and the present angle:

$$
\mathrm{E}[\mathrm{g} 2] \mathrm{t}=\gamma \mathrm{E}[\mathrm{g} 2] \mathrm{t}-1+(1-\gamma) \mathrm{gt} 2
$$

set $\gamma$ to a similar value as the momentum term, around 0.9 . For clarity, we now rewrite our vanilla SGD update in terms of the parameter update vector $\Delta \theta t$ :

$$
\begin{aligned}
& \Delta \theta \mathrm{t}=-\eta \cdot \mathrm{gt}, \mathrm{i} \\
& \theta \mathrm{t}+1=\theta \mathrm{t}+\Delta \theta \mathrm{t}
\end{aligned}
$$

\section{ADAM}

Abbreviate to Adaptive Moment Estimation, is very important technique that figures versatile learning rates for every parameter. Notwithstanding putting away an exponentially rotting normal of past squared angles vt like Adadelta, Adam likewise keeps an exponentially rotting normal of past inclinations mt, like energy [20]:

$$
\begin{aligned}
& \mathrm{vt}=\beta 2 \mathrm{vt}-1+(1-\beta 2) \mathrm{g} \mathrm{t} 2 \\
& \mathrm{mt}=\beta 1 \mathrm{mt}-1+(1-\beta 1) \mathrm{gt}
\end{aligned}
$$

Where $\mathrm{mt}$ and $\mathrm{vt}$ are assessments of the main minute (the mean) and the subsequent minute (the uncentered change) of the slopes separately, henceforth the name of the technique. As mt and vt are instated as vectors of 0 's, the creators of Adam see that they are one-sided towards zero, particularly during the underlying time steps, and particularly when the rot rates are little and close to 1 ( $\beta 1$ and $\beta 2$ ). They balance these predispositions by registering inclination redressed the minutes of first and second appraisals [21]:

$$
\begin{aligned}
& 1-\beta \mathrm{t} 1 \\
& \mathrm{~m}^{\wedge} \mathrm{t}=\mathrm{mt} / 1-\beta \mathrm{t} 1 \\
& \mathrm{v}^{\wedge} \mathrm{t}=\mathrm{vt} 1-\beta \mathrm{t} 2
\end{aligned}
$$




\section{PROPOSED SYSTEM}

This research focuses on create deep learning model based on CNN technique to make training, recognition and then classification to fingerprint, using Kaggle dataset consists from 80 print for 8 persons, ten prints to each one, Figure 1 shows the diagram of this model:

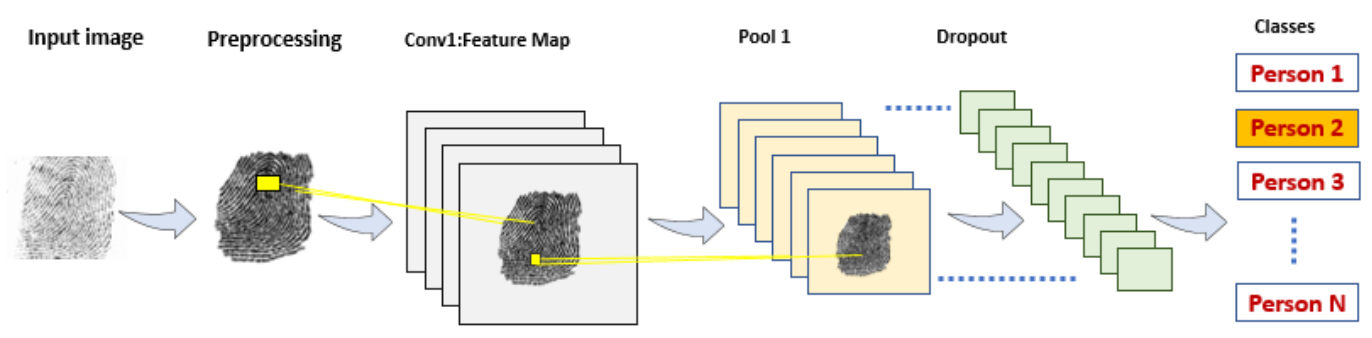

Figure 1. Model diagram

In this model, four Convolutional layers are used with depth (3X3X32,3X3X64,3X3X128, $3 \mathrm{X} 3 \mathrm{X} 128)$ sequentially, and each one is followed by max polling layers with filter size (2X2) for each one, and then drop out of $(0,3)$ is used.

\section{DATASET}

The third international fingerprint verification competition "FVC2004 DB1_B" dataset is utilized in this research [22], it collected in The Biometric Systems Lab (University of Bologna), the Pattern Recognition and Image Processing Laboratory (Michigan State University) and the Test Center (San Jose State University), each image size is 640x480 (307 Kpixels) scanned by optical sensor "V300" by CrossMatch with resolution 500dpi.

First of all, the model starts with loading the dataset, which is classed to 5 identities, each identity has ten prints for the same finger, by using keras's "ImageDataGeneratorlibrary" class, new data generated to slightly augment the data with shifts, rotations, zooms, and mirroring, Figure 2 shows sample of augmented dataset.

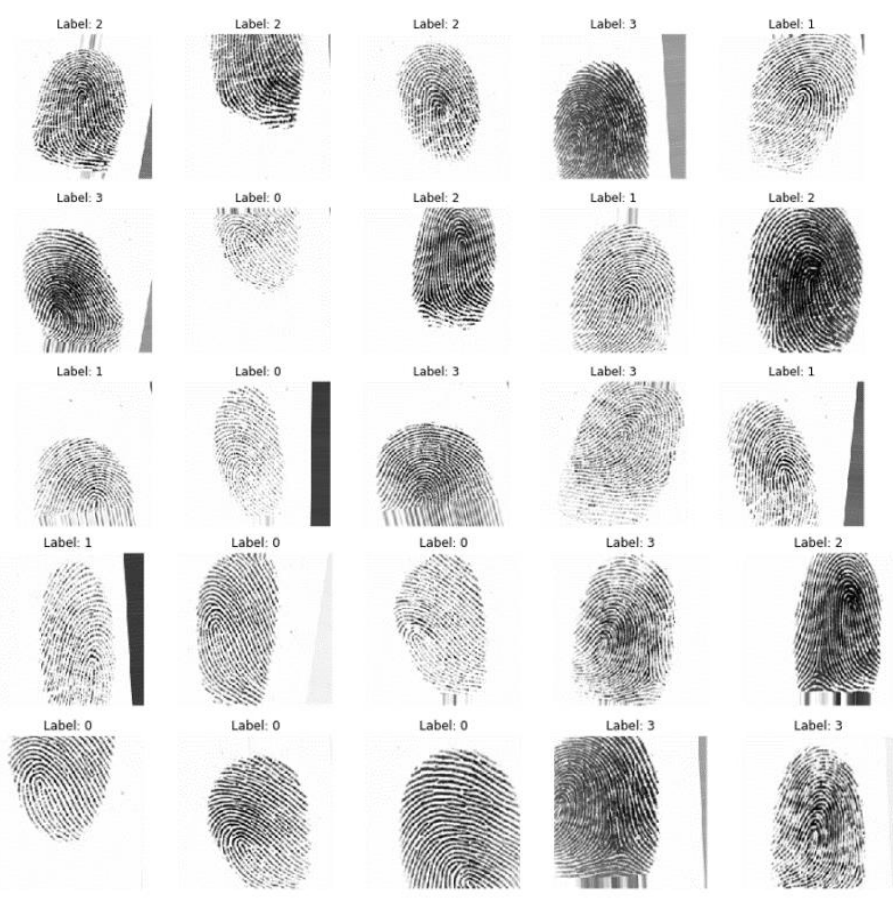

Figure 2. Augmented dataset 


\section{RESULTS AND DISCUSSION}

In this research, the code in [23] is depended as reference with some modifications in size of images in dataset and in the number of classes, which is considered as five instead of ten classes, to be compatible with fingerprint application, and the modification codes of this research is in [24, 25].

\subsection{Scenario 1}

In this scenario, using "Adadelta" Optimizer in CNN Model, without using histogram equalization to see the effect of "Adadelta" without enhancing in contrast, the training epochs are 11/40 with testing accuracy $=85.61 \%$, Figure 3 (a) shows the accuracy with epochs of training and testing operations.

\subsection{Scenario 2}

The Optimizer is changed with "Adam", Figure 3(b) shows the accuracy testing without using histogram equalization, the training epochs are $40 / 40$ with testing accuracy $=91.73 \%$.

\subsection{Scenario 3}

In this scenario, histogram equalization is used to enhance the contrast of fingerprint, with "Adadelta" Optimizer, where the training epochs are $14 / 40$ and the testing accuracy $=61.84 \%$ Figure 3(c) shows the accuracy testing in this scenario.

\subsection{Scenario 4}

In this scenario, histogram equalization is used to enhance the contrast of fingerprint, with "Adam" Optimizer, where the training epochs are 14/40 and the testing accuracy $=37.24 \%$ Figure 3(c) shows the accuracy testing in this scenario.

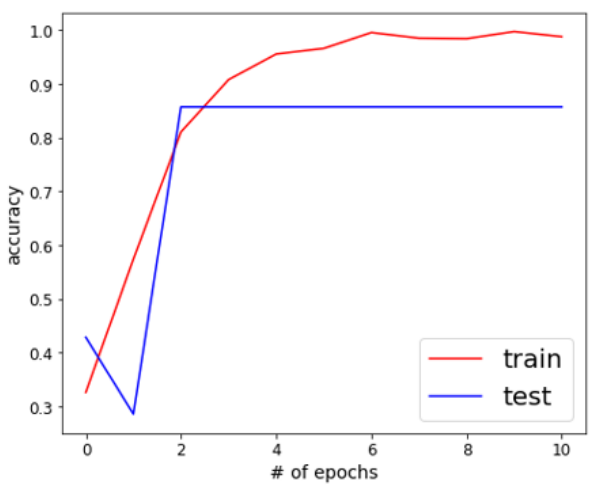

(a)

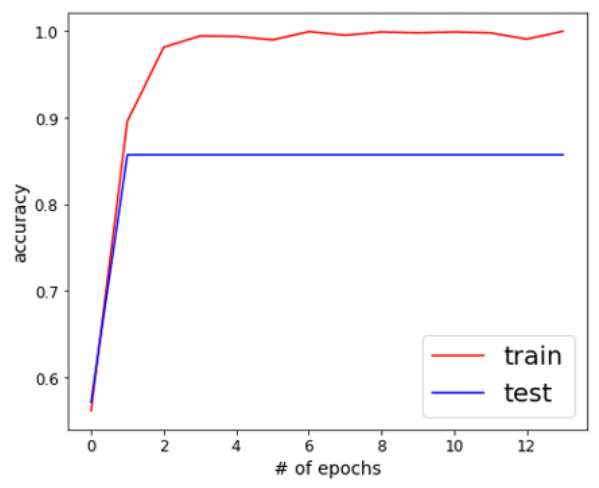

(c)

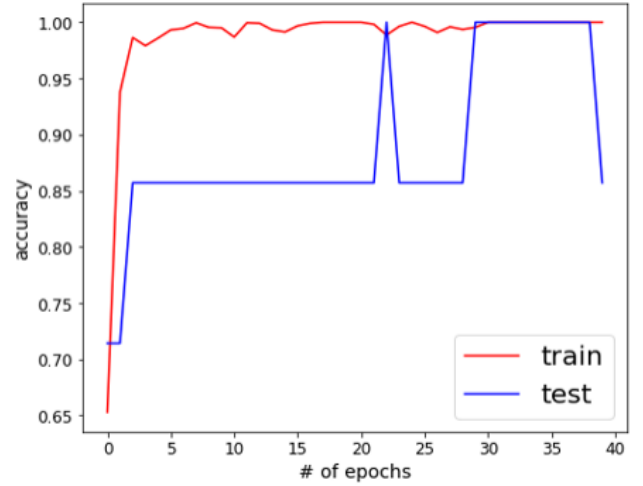

(b)

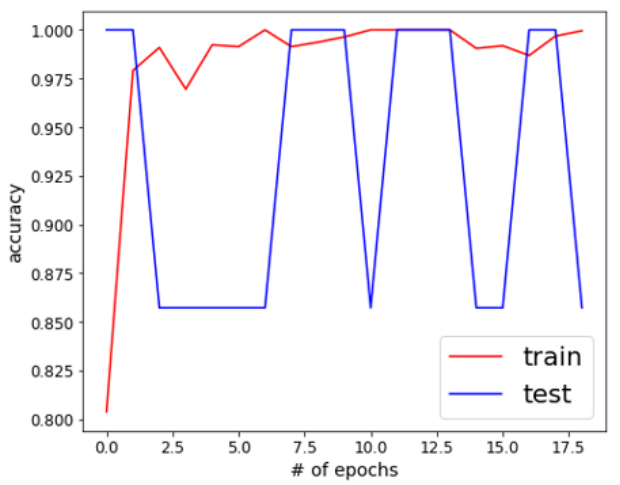

(d)

Figure 3. the accuracy with epochs of training and testing operations

\section{CONCLUSION}

The model is implemented and tested, CNN algorithm is used in proposed model and experiment in four scenarios, first one with "adadelta" Optimizer and the testing accuracy $=85.61 \%$, while the second 
scenario when used "Adam" Optimizer, the testing accuracy $=91.73 \%$, as a result, "Adam" Optimizer is more efficient than "adadelta" in fingerprint classification application, in addition, the third and fourth scenarios show that after using histogram equalization algorithm in fingerprint application does not enhance the fingerprints for classification as appears in the enhanced image where the contrast of image become sharpener with high contrast, but it does not increase the accuracy of classification where the accuracy was $61.84 \%$ and 37.24\% with "Adadelta" and "Adam" Optimizer.

\section{ACKNOWLEDGEMENTS}

The author would like to express the deepest appreciation and grateful to assistant professor "Dr. Shibly Ahmed Hammed" the head of Control Engineering branch, in University of Technology-Control and Systems engineering department for his assistant in check the similarity report of this research.

\section{REFERENCES}

[1] Zhukov, A.V.; Sidorov, D.N.; Foley, A.M. "Random forest based approach for concept drift handling," In Analysis of Images, Social Networks and Texts, Springer: Berlin, Germany, pp. 69-77, 2016.

[2] Wong, Wei Jing, and Shang-Hong Lai. "Multi-task CNN for restoring corrupted fingerprint images," Pattern Recognition, vol. 101, 2020.

[3] Hammad, Mohamed, and Kuanquan Wang. "Parallel score fusion of ECG and fingerprint for human authentication based on convolution neural network," Computers \& Security, vol. 81, pp. 107-122, 2019.

[4] Imane Hachchane, Abdelmajid Badri, et al., "Large-scale image-to-video face retrieval with convolutional neural network features," International Journal of Artificial Intelligence (IJ-AI) vol. 9, no. 1, pp. 40-45, 2020.

[5] Liu, Y., Zhou, B., Han, C., et al., "A novel method based on deep learning for aligned fingerprints matching," Appl Intell, vol. 50, no. 2, pp. 397-416, 2020.

[6] Huong Thu Nguyen, Long The Nguyen, "Fingerprints classification through image analysis and machine learning method, algorithms," Algorithms, vol. 12, no. 11, pp. 241, 2019.

[7] Sarker G., Ghosh S. "Biometric-based unimodal and multimodal person identification with CNN using optimal filter set”, In: Mandal J., Mukhopadhyay S. (eds) Proceedings of the Global AI Congress 2019. Advances in Intelligent Systems and Computing, vol 1112. Springer, Singapore, 2020.

[8] Zhang, Q.; Yan, H. "Fingerprint classification based on extraction and analysis of singularities and pseudo ridges," Pattern Recognit, vol. 37, no. 11, pp. 2233-2243, 2004.

[9] Hong, J. H., Min, J. K., Cho, U. K., "Fingerprint classification using one-vs-all support vector machines dynamically ordered with Bayes classifiers," Pattern Recognit, vol. 41, no. 2, pp. 662-671, 2008.

[10] Senior, A., "A combination fingerprint classifier," IEEE Trans. Pattern Anal. Mach. Intell., vol. 23, no. 10, pp. 1165-1174, 2001.

[11] Karu, K. A Jain, "Fingerprint Classification," Pattern Recognition, vol. 29, no. 3, pp. 389-404, 1996.

[12] Nyongesa, H.; Al-khayatt, S., "Fast robust fingerprint feature extraction and classification," Journal of Intelligent and Robotic Systems, vol. 40, no. 1, pp. 103-112, 2004.

[13] Nagaty, K. "Fingerprints classification using artificial neural networks: A combined structural and statistical approach," Neural Networks, vol. 14, no. 9, pp. 1293-1305, 2001.

[14] Guo, T.; Liu, X.; Shao, G., "Fingerprint classification based on sparse representation using rotation-invariant features," In Proceedings of the 2012 International Conference on Information Technology and Software Engineering, Springer: Berlin/Heidelberg, Germnay, pp. 631-641, 2013.

[15] Schroff, F.; Criminisi, A.; Zisserman, A., "Object class segmentation using randomforests," In Proceedings of the British Machine Vision Conference 2008, Leeds, UK, pp. 1-10, 2008.

[16] Jadhav, Sachin B. "Convolutional neural networks for leaf image-based plant disease classification," International Journal of Artificial Intelligence (IJ-AI), vol. 8, no. 4, pp. 328, 2019.

[17] El mehdi Cherrat, Rachid Alaoui, and Hassane Bouzahir. "A multimodal biometric identification system based on cascade advanced of fingerprint, fingervein and face images," Indonesian Journal of Electrical Engineering and Computer Science (IJEECS), vol. 18, no. 1, 1562-1570, 2020.

[18] Fairuz, Subha, Mohamed Hadi Habaebi, and Elsheikh Mohamed Ahmed Elsheikh, "Pre-trained based CNN model to identify finger vein," Bulletin of Electrical Engineering and Informatics (BEEI), vol. 8, no. 3, pp. 855-862, 2019.

[19] Matthew D. Zeiler, "ADADELTA: An Adaptive Learning Rate Method," arXiv preprint arXiv:1212.5701, 2012.

[20] D. P. Kingma and J. Ba, "Adam: A method for stochastic optimization," arXiv preprint arXiv:1412.6980, 2014.

[21] Diederik P. Kingma and Jimmy Lei Ba, "Adam: A method for stochastic optimization," International Conference on Learning Representations, pp. 1-13, 2015.

[22] D. Maltoni, D. Maio, A.K. Jain, S. Prabhakar, "The full FVC2000, FVC2002, and FVC2004 databases are available in the DVD included" in: Handbook of Fingerprint Recognition (Second Edition), London: Springer, 2009. [Online]. Available: http://bias.csr.unibo.it/fvc2004/download.asp.

[23] "CNN-HowManyFingers," 2017, [Online]. Available: https://github.com/jaredvasquez/CNN-HowManyFingers.

[24] [Online]. Available: Fingerprint"https://drive.google.com/open?id=1u3KhX1R49CVj58GaYrnFlbD_7qEVcmcT.

[25] "Optimizers," Keras, [Online]. Available: https://keras.io/optimizers/ 